\title{
Uso de milrinona no tratamento da hipertensão pulmonar persistente do recém-nascido
}

\author{
Milrinone for persistent pulmonary hypertension of the newborn treatment
}

Gisela de Rezende Eugênio ${ }^{1}$, Flávia Carolina D. Georgetti

\section{RESUMO}

Objetivo: Descrever uma série de casos de recém-nascidos com hipertensão pulmonar persistente grave, que receberam milrinona para promover a vasodilatação pulmonar.

Métodos: Análise retrospectiva de prontuários de 28 pacientes com diagnóstico de hipertensão pulmonar persistente do recém-nascido (HPPRN). Após o diagnóstico, todos os pacientes receberam uma dose de ataque de $50 \mathrm{mcg} / \mathrm{kg}$ de milrinona, seguida por $0,75 \mathrm{mcg} / \mathrm{kg} / \mathrm{min}$. O índice de oxigenação (IO) foi calculado no início da infusão e 72 horas após o início da medicação.

Resultados: Todos os neonatos receberam milrinona e o sildenafil foi associado em 54\%. O uso de dopamina assegurou a manutenção da pressão arterial em nível adequado em todos os casos. Sedação contínua, alcalinização e surfactante foram medidas coadjuvantes no tratamento. Durante a internação, sete pacientes $(25 \%)$ evoluíram a óbito e todos eles apresentaram aumento do IO, com elevação da média de 25 para 38 com a milrinona. Os sobreviventes, com exceção de um neonato, apresentaram redução do $\mathrm{IO}$ em uso de milrinona, com queda da média de 19 para 7 .

Conclusões: $\mathrm{O}$ uso da milrinona parece ser uma alternativa para o tratamento da HPPRN, na ausência do óxido nítrico. A redução do $\mathrm{IO}$ com a medicação foi fator determinante da boa evolução dos pacientes. $\mathrm{O}$ índice de falha no tratamento com a milrinona nesta casuística foi semelhante ao encontrado na literatura para o uso de óxido nítrico.

Palavras-chave: síndrome da persistência do padrão de circulação fetal; inibidores de fosfodiesterase; milrinona.

${ }^{1}$ Coordenadora da Unidade de Terapia Intensiva Neonatal (UTIN) dos Hospitais Santa Elisa e Universitário da Faculdade de Medicina de Jundiaí (FMJ). Professora colaboradora do Departamento de Pediatria da FMJ, Jundiaí, SP, Brasil

${ }^{2}$ Coordenadora da UTIN do Hospital Santa Elisa e professora colaboradora do Departamento de Pediatria da FMJ, Jundiaí, SP, Brasil

\section{ABSTRACT}

Objective: To describe a series of neonates with severe persistent pulmonary hypertension, who received milrinone as the main treatment for pulmonary vasodilatation.

Methods: Retrospective analysis by chart review of 28 neonates with persistent pulmonary hypertension. A dose of $0.75 \mu \mathrm{g} / \mathrm{kg} / \mathrm{min}$ of milrinone was given, after a loading dose of $50 \mu \mathrm{g} / \mathrm{kg}$. The oxygenation index (OI) was calculated before and 72 hours after the medication.

Results: All infants received milrinone and sildenafil was associated to milrinone in $54 \%$. The use of dopamine assured normal blood pressure during milrinone treatment in all patients. Continuous sedation, alcalinization and surfactant were additional measures in the treatment. During the hospitalization period, seven (25\%) patients died and all of them presented an OI increase after milrinone (the average OI rose from 25 to 38). All but one of the 21 surviving patients presented improvement of the OI with milrinone, with a reduction of the mean index from 19 to 7 .

Conclusions: Milrinone can be used to treat persistent pulmonary hypertension of the newborn, in the absence of nitric oxide. The reduction of the OI during treatment was associated with clinical improvement. The failure rate for milrinone treatment in this series of cases was similar to that found in the literature regarding nitric oxide.

Key-words: persistent fetal circulation syndrome; phosphodiesterase inhibitors; milrinone.
Endereço para correspondência:

Gisela de Rezende Eugênio

Rua Avelina Corrêa de Paula, 110

Condomínio Portal do Paraíso 1

CEP 13214-671 - Jundiaí/SP

E-mail: gr-eugenio@uol.com.br. 


\section{Introdução}

A hipertensão pulmonar persistente do recém-nascido (HPPRN) é uma doença complexa, caracterizada pela presença de hipoxemia grave logo após o nascimento, hipertensão pulmonar intensa e vasorreatividade pulmonar, com shunt de sangue extrapulmonar da direita para a esquerda, através do canal arterial e/ou forame oval, na ausência de cardiopatia congênita cianogênica ${ }^{(1)}$.

A HPPRN é uma doença relativamente comum em recém-nascidos a termo ou próximo do termo, com incidência de 0,43 a 6,8/1.000 nascidos vivos, associada a altos índices de morbidade, sobretudo pelo dano neurológico em 15 a $20 \%$ dos casos, e mortalidade de 10 a $20 \%$, a despeito de todos os avanços diagnósticos e terapêuticos ${ }^{(1-4)}$.

$\mathrm{O}$ atraso ou a incapacidade de adaptação do neonato à alteração do padrão de circulação fetal para neonatal é responsável pelo desenvolvimento do quadro de HPPRN. A imaturidade dos vasos pulmonares decorrente de hipoplasia pulmonar, como nos casos de hérnia diafragmática congênita, o mau desenvolvimento dos vasos pulmonares, como nos casos de uso materno prolongado de aspirina ou indometacina, ou ainda a não adaptação funcional dos vasos pulmonares são os principais fatores responsáveis pelo desenvolvimento do quadro.

O conceito de má adaptação consiste em falha na queda da resistência vascular pulmonar logo após o nascimento, que ocorre na presença de anatomia pulmonar normal. A falha no mecanismo de dilatação das artérias pulmonares, responsável pela redução da resistência vascular pulmonar, pode ser induzida pela presença de hipoxemia, acidose, aspiração pulmonar de sangue ou mecônio, entre outros. Os pacientes portadores de HPPRN decorrente de alterações perinatais, que levam à doença vasoespástica potencialmente reversível, são considerados excelentes candidatos ao tratamento do quadro com vasodilatadores pulmonares ${ }^{(5)}$.

O tratamento dos bebês com HPPRN tem por objetivo minimizar a resistência vascular pulmonar, otimizando o fluxo sanguíneo pulmonar, sem que ocorra prejuízo do débito cardíaco. Nos últimos anos, muitos agentes vasodilatadores têm sido utilizados como coadjuvantes no tratamento dos recém-nascidos com hipertensão pulmonar reativa em associação à assistência ventilatória e à correção dos distúrbios de base. Entretanto, com exceção do óxido nítrico, as demais terapias vasodilatadoras são de uso limitado por não agirem exclusivamente na circulação pulmonar.

Durante muitos anos, o óxido nítrico, um vasodilatador pulmonar seletivo, foi considerado o método de tratamento padrão-ouro capaz de promover a redução da resistência vascular pulmonar nos neonatos com HPPRN grave, sendo indicado, sobretudo, nos casos em que o índice de oxigenação fosse maior ou igual a $25^{(6)}$. Embora estudos randomizados, realizados em pacientes com HPPRN tratados com óxido nítrico tenham falhado em demonstrar significativa redução no tempo de internação e, sobretudo, nos índices de mortalidade, o uso desse vasodilatador evitou a necessidade de oxigenação por membrana extracorpórea $(\mathrm{ECMO})^{(7-12)}$.

Entretanto, além da possibilidade de falha terapêutica, estimada em até $30 \%$, o custo do óxido nítrico e da ECMO são bastante elevados, principalmente nos países em desenvolvimento ${ }^{(13)}$. Neste contexto, a necessidade de encontrar tratamentos alternativos ao uso de óxido nítrico e ECMO possibilitou a tentativa de aplicação de novos vasodilatadores pulmonares neste pacientes. Nos últimos anos, os inibidores de fosfodiesterase têm sido investigados como potenciais coadjuvantes na melhora da oxigenação em pacientes com HPPRN grave. O objetivo deste estudo é descrever uma série de casos de recém-nascidos com HPPRN grave, internados em um centro em que não havia disponibilidade para o uso de óxido nítrico e que receberam milrinona, um inibidor da fosfodiesterase III, como principal medida para vasodilatação pulmonar.

\section{Métodos}

Os dados apresentados neste estudo foram obtidos por meio da análise retrospectiva dos prontuários de 29 neonatos com diagnóstico de hipertensão pulmonar persistente, internados na Unidade de Terapia Intensiva Neonatal (UTIN) do Hospital Santa Elisa, em Jundiaí, São Paulo, após a aprovação pelo Comitê de Ética da instituição. Recém-nascidos que evoluíram com cianose progressiva nas primeiras 12 a 24 horas de vida foram submetidos à avaliação da saturação de oxigênio pré e pós-ductal. Uma vez verificado o diferencial de $10 \%$ ou mais, os neonatos foram submetidos à realização de ecocardiograma bidimensional para confirmar o diagnóstico de HPPRN. O índice de oxigenação, parâmetro utilizado na avaliação de resposta ao milrinona, foi calculado imediatamente antes do início da medicação e após 72 horas, por meio da fórmula: pressão média de vias aéreas multiplicada pela fração inspirada de oxigênio, dividida pela pressão parcial arterial de oxigênio, multiplicada por $100\left(\mathrm{PMVAxFiO}_{2} / \mathrm{PaO}_{2} \mathrm{x} 100\right)$.

Para comparar os grupos de sobreviventes e não sobreviventes, utilizou-se, para variáveis numéricas com distribuição normal, o teste $t$ de Student e, para aquelas que não apresentavam distribuição normal, o teste de Mann-Whitney. 
Para as variáveis qualitativas, foram aplicados o teste do qui-quadrado e o exato de Fisher. Para analisar as medidas repetidas do índice de oxigenação inicial e com 72 horas, empregou-se o teste de Wilcoxon pareado.

Tabela 1 - Características demográficas da população estudada, segundo a evolução clínica

\begin{tabular}{lccc}
\hline Características & $\begin{array}{c}\text { Sobreviventes } \\
(\mathbf{n = 2 1 )}\end{array}$ & $\begin{array}{c}\text { Óbitos } \\
(\mathbf{n = 7})\end{array}$ & $\boldsymbol{p}$ \\
\hline Peso ao nascer $(\mathrm{g})$ & $3.040 \pm 494$ & $2.915 \pm 284$ & 0,54 \\
Idade gestacional (sem) & $37,2 \pm 2,1$ & $37,6 \pm 1,5$ & 0,65 \\
Prematuros & $7(33 \%)$ & $1(14 \%)$ & 0,33 \\
Cesariana & $17(81 \%)$ & $6(86 \%)$ & 0,63 \\
Apgar de 1 minuto $<7$ & $5(24 \%)$ & $4(57 \%)$ & 0,12 \\
Apgar de 5 minutos $\geq 7$ & $16(76 \%)$ & $3(43 \%)$ & 0,12 \\
Sexo masculino & $17(81 \%)$ & $4(75 \%)$ & 0,22 \\
\hline
\end{tabular}

Tabela 2 - Diagnósticos clínicos iniciais e evolutivos da população estudada

\begin{tabular}{lccc}
\hline Diagnósticos iniciais & $\begin{array}{c}\text { Sobreviventes } \\
(\mathbf{n = 2 1})\end{array}$ & $\begin{array}{c}\text { Óbitos } \\
(\mathbf{n}=7)\end{array}$ & $\boldsymbol{p}$ \\
\hline TTRN & $9(43 \%)$ & -- & \\
DMH & $8(38 \%)$ & $1(14 \%)$ & 0,25 \\
SAM & $1(5 \%)$ & $1(14 \%)$ & 0,44 \\
Pneumonia & $1(5 \%)$ & $2(29 \%)$ & 0,15 \\
Sepse precoce & $13(62 \%)$ & $4(57 \%)$ & 0,58 \\
Hemorragia & $1(5 \%)$ & --- & \\
pulmonar/CIVD & --- & $1(14 \%)$ & \\
Aspiração de sangue & --- & $1(14 \%)$ & \\
Hérnia diafragmática & $2(10 \%)$ & $4(57 \%)$ & 0,02 \\
Asfixia perinatal & & & \\
\hline Diagnósticos evolutivos & & $4(57 \%)$ & 0,18 \\
IRA & $6(29 \%)$ & $2(29 \%)$ & 0,58 \\
PCA & $5(24 \%)$ & $4(43 \%)$ & 0,59 \\
Sepse tardia & $10(48 \%)$ & 0,02 \\
Ganho de peso com 72 & 9 & 41 & \\
horas de vida (g) & & & \\
\hline
\end{tabular}

TTRN: taquipnéia transitória do RN; DMH: doença das membranas hialinas; SAM: síndrome da aspiração de mecônio; CIVD: coagulação intravascular disseminada; IRA: insuficiência renal aguda; PCA: persistência do canal arterial.

\section{Resultados}

No período de agosto de 2005 a fevereiro de 2007, nasceram vivos 2.719 pacientes no Hospital Santa Elisa e 29 foram internados na UTIN com o diagnóstico de HPPRN (1,06/1.000 nascidos vivos). Destes 29 pacientes identificados como potenciais portadores de HPPRN do ponto de vista clínico, 28 tiveram o diagnóstico confirmado pelo ecocardiograma. Esse exame excluiu a presença de cardiopatias congênitas em todos os casos.

Dos 28 neonatos estudados, 23 haviam nascido de cesárea e cinco de parto vaginal. Houve predominância do sexo masculino (21 recém-nascidos, $75 \%$ ). A média de peso ao nascimento foi $3.009 \mathrm{~g}$, com variação de $1.920 \mathrm{~g}$ a $3.840 \mathrm{~g}$, e a idade gestacional foi 37 semanas e três dias (variação de $33^{6 / 7}$ a $41^{5 / 7}$ ). Em oito neonatos, o Apgar no primeiro minuto foi $\leq 5$ e cinco mantiveram Apgar $\leq 7$ no quinto minuto de vida (Tabela 1).

No momento do parto, não foram identificados fatores de risco para o desenvolvimento de HPPRN em sete casos (cesáreas eletivas). Nos demais 21 neonatos, havia risco para sepse neonatal em 11, sendo que nove mães apresentavam bolsa rota por tempo superior a 18 horas e duas estavam em tratamento de infecção urinária. Sofrimento fetal ao ultrasom foi indicação da interrupção da gestação em quatro pacientes; duas gestantes apresentaram sangramento vaginal e outras três, doença hipertensiva específica da gestação; em um caso, houve descolamento prematuro de placenta e em outro havia o diagnóstico ultra-sonográfico de hérnia diafragmática congênita.

Após o nascimento, os diagnósticos iniciais foram taquipnéia transitória do recém-nascido, doença de membrana hialina, síndrome de aspiração de mecônio, pneumonia intra-útero, aspiração maciça de sangue, hemorragia pulmonar, hérnia diafragmática congênita e asfixia perinatal, conforme Tabela 2.

Uma vez diagnosticado o quadro de HPPRN, os pacientes foram submetidos a um regime de cuidados sob manipulação mínima e receberam assistência ventilatória sob sedação e analgesia contínua com midazolam e fentanil. Nenhum recebeu bloqueador neuromuscular. Para os neonatos nos quais a causa da HPPRN esteve relacionada à doença pulmonar parenquimatosa, indicou-se reposição de surfactante pulmonar exógeno. Em 17 recém-nascidos, foi utilizado o bicarbonato de sódio com o objetivo de promover alcalinização. Os quadros de infecção foram conduzidos com antibioticoterapia. Os pacientes que apresentaram choque 
foram avaliados individualmente e tratados com o uso de cristalóides e de drogas vasoativas, de acordo com a necessidade. O uso de hemocomponentes (concentrado de hemácias, plasma fresco congelado e/ou plaquetas) foi necessário em $46 \%$ dos pacientes.

A milrinona, como vasodilatador pulmonar, foi indicada para todos os recém-nascidos que mantiveram a necessidade de $\mathrm{FiO}_{2}$ maior ou igual a 0,8 sob assistência ventilatória, a despeito de todas as medidas anteriormente descritas, em decorrência da indisponibilidade de óxido nítrico em nosso hospital. A dose utilizada foi $0,75 \mathrm{mcg} / \mathrm{kg} / \mathrm{min}$ após uma dose de ataque de $50 \mathrm{mcg} / \mathrm{kg}$. O uso de dopamina, associada à infusão de milrinona, assegurou a manutenção da pressão arterial em níveis adequados em todos os pacientes. O tempo médio em uso de milrinona foi 6 dias. O esquema de retirada da medicação consistiu na redução de $25 \%$ da dose a cada 12 horas, após alcançar $\mathrm{PaO}_{2}$ acima de $70 \mathrm{mmHg}$ em uso de $\mathrm{FiO}_{2}$ igual a 0,5 (Tabela 3).

Para os pacientes nos quais não foi possível reduzir a pressão média de vias aéreas após 48 horas de uso da milrinona, administrou-se sildenafil na dose de $2 \mathrm{mg} / \mathrm{kg}$ a cada seis horas. Apenas no paciente com hérnia diafragmática congênita, esta medicação foi associada à milrinona desde o início do tratamento. O paciente, no entanto, evoluiu para óbito no terceiro dia de vida (Tabela 3).

Durante a internação, sete neonatos apresentaram sinais clínicos de persistência de canal arterial, após resolução do quadro de hipertensão pulmonar. Uma vez confirmado o diagnóstico pela ecocardiografia, os pacientes receberam tratamento com ibuprofeno por via enteral e, em todos, houve o fechamento do canal arterial (Tabela 3).

Dos 28 neonatos analisados neste estudo, 23 apresentaram alguma anormalidade no débito urinário e receberam furosemide. Destes, cinco pacientes evoluíram com insuficiência renal grave e necessidade de diálise peritoneal, sendo constatado o óbito de quatro deles durante a evolução (Tabela 3).

O tempo médio sob ventilação pulmonar mecânica para os sobreviventes foi de dez dias. Durante a internação, sete pacientes evoluíram a óbito (25\%), sendo que dois apresentavam pneumonia intra-útero; um havia sido internado em decorrência de asfixia perinatal grave e um apresentava hérnia diafragmática. A síndrome de aspiração meconial, a doença de membrana hialina e a aspiração maciça de sangue foram responsáveis pela internação dos demais pacientes.

Todos os neonatos que evoluíram a óbito apresentaram elevação no índice de oxigenação após 72 horas, sendo observado um aumento na média deste índice de 24,65 no inicio da infusão de milrinona para 38,2 após 72 horas de uso da droga $(p<0,001)$. Entre os sobreviventes, no entanto, foi observada uma redução no índice de oxigenação após 72 horas do início da infusão de milrinona, com queda da média do índice de 19,2 para 6,6 ( $p=0,016)$, conforme Tabela 4 . A presença de acidose metabólica, representada pela necessidade do uso de alcalinização $(p=0,016)$ e a ocorrência de asfixia perinatal $(p=0,020)$ se associaram ao mau prognóstico.

\section{Discussão}

O diagnóstico de hipertensão pulmonar persistente deve ser considerado em um neonato que evolui com cianose progressiva nas primeiras 12 a 24 horas de vida, algumas vezes após um período de oxigenação adequada, e/ou que demonstra labilidade na oxigenação. $\mathrm{O}$ diagnóstico baseiase no reconhecimento de possíveis fatores de risco para o desenvolvimento da doença, no exame físico (com especial atenção aos sinais de desconforto respiratório e cianose), no raio-X de tórax (cardiomegalia e diminuição da vasculatura

Tabela 3 - Comparação dos grupos de sobreviventes e não sobreviventes em relação ao tratamento instituído

\begin{tabular}{lccc}
\hline Tratamento & $\begin{array}{c}\text { Sobreviventes } \\
(\mathbf{n = 2 1 )}\end{array}$ & $\begin{array}{c}\text { Óbitos } \\
(\mathbf{n = 7})\end{array}$ & $\boldsymbol{p}$ \\
\hline Surfactante & $18(86 \%)$ & $7(100 \%)$ & 0,41 \\
Alcalinização & $10(48 \%)$ & $7(100 \%)$ & 0,02 \\
Sildenafil & $10(48 \%)$ & $5(71 \%)$ & 0,22 \\
lbuprofeno & $5(24 \%)$ & $1(14 \%)$ & 0,52 \\
Dobutamina & $9(43 \%)$ & $5(71 \%)$ & 0,19 \\
Furosemida & $16(76 \%)$ & $7(100 \%)$ & 0,21 \\
Diálise peritonial & $1(5 \%)$ & $4(57 \%)$ & 0,04 \\
Dias de ventilação* & 10 & 10 & 0,32 \\
Dias de internação* & 19 & 10 & 0,13 \\
\hline *
\end{tabular}

* expresso em média

Tabela 4 - Comparação dos grupos de sobreviventes e não sobreviventes em relação ao índice de oxigenação após o início do uso de milrinona, expresso em média (variação)

\begin{tabular}{lcc}
\hline & Sobreviventes & Óbitos \\
\hline IO inicial & 19,2 & 24,7 \\
& $(8,5-30,6)$ & $(13,3-40,5)$ \\
IO 72 horas & 6,6 & 38,2 \\
& $(1,4-34,7)$ & $(15,1-68,7)$ \\
valor de $p$ & 0,015 & $<0,001$ \\
\hline
\end{tabular}


pulmonar ou opacidades pulmonares, em caso de pneumonia intra-útero ou síndromes aspirativas), no diferencial de $15 \mathrm{mmHg}$ ou mais na pressão arterial de oxigênio avaliada pelas gasometrias pré e pós-ductal e no ecocardiograma (capaz de demonstrar o aumento da pressão na artéria pulmonar, o shunt da direita para a esquerda através do canal arterial ou do forame oval e, algumas vezes, regurgitação na válvula tricúspide). O hemograma e outros marcadores de sepse, como a proteína $C$ reativa, podem ser necessários para confirmar o diagnóstico de infecção.

$\mathrm{O}$ tratamento consiste na tentativa de controle do fator desencadeante da HPPRN e na instituição de medidas que possam diminuir a resistência vascular pulmonar, além de assegurar a adequada liberação de oxigênio aos tecidos. A manutenção da pressão arterial sistêmica e a minimização da lesão pulmonar induzida pela ventilação são medidas essenciais ao sucesso do tratamento destes pacientes.

O neonato deve ser acomodado em um ambiente termorregulado, sedado e submetido a um regime de cuidados sob manipulação mínima. Com muita freqüência, sedativos e opióides são utilizados para o conforto desses pacientes. O uso de bloqueadores neuromusculares, como pancurônio, vem sendo desencorajado em decorrência de um possível aumento do risco de óbito ${ }^{(14)}$. A infusão de cristalóides e a de agentes inotrópicos e vasopressores é muitas vezes imprescindível para manter a pressão arterial em níveis adequados em pacientes com HPPRN grave. Os antibióticos, por sua vez, são essenciais nos casos de infecção.

O surfactante pulmonar exógeno tem sido relacionado à diminuição do risco de lesão pulmonar, de síndrome de escape de ar e da duração do período de internação em pacientes com HPPRN decorrente de doença da membrana hialina, hérnia diafragmática, síndrome da aspiração meconial e/ou pneumonia intra-útero, situações nas quais a estabilização do volume alveolar é fundamental ${ }^{(5,15-16)}$.

A ventilação pulmonar mecânica, que visa assegurar a oxigenação adequada aos tecidos, é uma das principais estratégias utilizadas no tratamento da HPPRN. Durante muito tempo, a hiperventilação, com o objetivo de aumentar o pH sanguíneo, reverter o shunt ductal e induzir a vasodilatação pulmonar, foi utilizada na ventilação pulmonar convencional. Entretanto, tal estratégia foi abandonada, pois a alcalose respiratória parece se associar ao aumento de lesão neurológica, com leucomalácia periventricular e paralisia cerebral, mesmo em recém-nascidos a termo ${ }^{(17)}$. A utilização de picos de pressão menos elevados, para alcançar valores de $\mathrm{pH}$ entre 7.4 e 7.5, níveis de $\mathrm{PaCO}_{2}$ entre 40 e
$60 \mathrm{mmHg}$ e a manutenção da $\mathrm{PaO}_{2}$ entre 60 e $90 \mathrm{mmHg}$ podem minimizar a lesão pulmonar ${ }^{(17-19)}$.

A ventilação de alta freqüência tem sido recomendada como a melhor estratégia de assistência ventilatória para os portadores de HPPRN decorrente de doença parenquimatosa pulmonar. A ventilação de alta freqüência parece otimizar a expansão pulmonar, ao mesmo tempo em que minimiza a lesão pulmonar por reduzir os traumas decorrentes do volume e da pressão $0^{(20-21)}$. Este tipo de ventilação parece melhorar a resposta ao óxido nítrico inalado, devido à otimização do recrutamento alveolar ${ }^{(22-23)}$

O óxido nítrico, um vasodilatador pulmonar potente e seletivo, é considerado o tratamento de escolha para a HPPRN grave. A substância é produzida endogenamente na célula endotelial e age pelas vias do AMP e GMP cíclicos, mediadas pelos canais de cálcio e potássio. Entretanto, nos neonatos com HPPRN, a ativação do sistema da endotelina resulta na diminuição da síntese de óxido nítrico endógeno. O seu uso está indicado em pacientes que apresentem idade gestacional superior a 34 semanas e índice de oxigenação maior do que 25 ou $\mathrm{PaO}_{2}<100 \mathrm{mmHg}$ sob $\mathrm{FiO}_{2}$ de $1.0^{(24)}$. A dose recomendada para administração por via inalatória varia entre 5 e $20 \mathrm{ppm}$. Uma vez que o óxido nítrico age quase que exclusivamente sobre os vasos pulmonares, os efeitos colaterais são mínimos, com praticamente nenhuma interferência sobre a pressão arterial sistêmica. $\mathrm{O}$ uso de doses elevadas do gás pode resultar em toxicidade decorrente do acúmulo de metahemoglobina. O possível efeito rebote de hipoxemia, no momento da retirada do óxido nítrico, pode ser minimizado pela associação de outros vasodilatadores pulmonares, como os inibidores de fosfodiesterase. A ECMO é indicada nos casos de insucesso quanto à melhora da oxigenação, em pacientes com HPPRN que estão recebendo óxido nítrico.

O sulfato de magnésio, um antagonista do cálcio que atua na contração das células musculares, administrado na dose de ataque de $200 \mathrm{mg} / \mathrm{kg}$, com manutenção de 30 a $150 \mathrm{mg} / \mathrm{kg} / \mathrm{h}$, é uma alternativa de baixo custo para tratar neonatos com HPPRN em locais onde o óxido nítrico não é disponível ${ }^{(25-27)}$. $\mathrm{O}$ uso do sulfato de magnésio pode, no entanto, determinar redução na pressão arterial sistêmica, sendo muitas vezes necessária a infusão simultânea de dopamina.

$\mathrm{Na}$ década de 1990, Patole $e t a^{(28)}$ demonstraram que a infusão de adenosina, na dose de 25 a $50 \mathrm{mcg} / \mathrm{kg} / \mathrm{min}$, melhorava a oxigenação de pacientes com HPPRN, mas que a ocorrência de graves efeitos adversos, como bradicardia, hipotensão arterial e aumento do tempo de sangramento, 
seria um importante fator limitante para seu uso. Posteriormente, $\mathrm{Ng}$ et al ${ }^{(29)}$ demonstraram que a associação de dopamina, adenosina e óxido nítrico determina um aumento na oxigenação, com manutenção adequada de pressão arterial sistêmica.

Nos últimos anos, os inibidores de fosfodiesterase (PDEs), enzimas que catalisam a clivagem hidrolítica dos nucleotídeos cíclicos, assumiram um importante papel no tratamento da HPPRN. Existem 11 tipos de PDEs distribuídos de maneira específica nos tecidos. As isoenzimas PDE3 e PDE5 são as predominantemente encontradas nos vasos pulmonares e tornaram-se potenciais alvos para o tratamento farmacológico da HPPRN. A milrinona é um inibidor seletivo da PDE3, presente nos miócitos cardíacos e na musculatura lisa pulmonar. Na década de 1990, estudos experimentais já demonstravam o possível efeito da milrinona na redução da resistência vascular pulmonar, em modelos animais de hipertensão pulmonar ${ }^{(30)}$. Na mesma época, a milrinona foi empregada como coadjuvante no tratamento da hipertensão pulmonar de neonatos em pós-operatório de correção de cardiopatias congênitas ${ }^{(31)}$. McNamara et al e Bassler et al descreveram duas séries de recém-nascidos com HPPRN não responsivos ao uso de óxido nítrico, que obtiveram melhora na oxigenação, sem prejuízo na pressão arterial sistêmica, após o uso da milrinona ${ }^{(32,33)}$.

O sildenafil, um inibidor bastante seletivo da PDE5, tem sido apontado como a chave de regulação da vasodilatação pulmonar induzida pelo óxido nítrico, sendo capaz de induzir vasodilatação pulmonar pela queda da resistência vascular pulmonar, com mínimo efeito sobre a circulação sistêmica $^{(34-38)}$. O mecanismo de ação do sildenafil consiste na inibição da PDE5, envolvida na degradação do GMP cíclico a GMP, resultando no aumento dos níveis intracelulares de GMPc e na conseqüente vasodilatação do leito pulmonar. A medicação deve ser administrada pela via enteral na dose de 0,5 a $2 \mathrm{mg} / \mathrm{kg}$, com intervalo de seis horas. Marsh et al ${ }^{(39)}$ relataram a possibilidade de retinopatia da prematuridade associada ao uso de sildenafil.

A milrinona e o sildenafil são vasodilatadores pulmonares potentes, com ações independentes e efeitos aditivos. Recentemente, Lobato et al demonstraram, em estudo experimental, que o uso concomitante das duas medicações determinou um aumento da contratilidade cardíaca, associado à vasodilatação pulmonar, sem interferência sobre a circulação sistêmica. Os autores observaram que, embora o sildenafil fosse capaz de determinar queda da resistência vascular pulmonar mais importante do que a produzida pela milrinona, esta última medicação foi particularmente responsável pela melhora do perfil hemodinâmico sistêmico, caracterizada pelo melhor desempenho do ventrículo direito, pelo aumento do débito cardíaco e pela preservação da resistência vascular sistêmica ${ }^{(40)}$.

No presente estudo, o uso dos inibidores da milrinona consistiu em alternativa para o tratamento da HPPRN grave, frente à indisponibilidade do óxido nítrico. A melhora na oxigenação, determinada pela queda do índice de oxigenação após 72 horas em uso da medicação, foi fator determinante da boa evolução dos pacientes. O uso de dopamina concomitante à infusão dos inibidores de fosfodiesterase assegurou a manutenção da pressão arterial sistêmica em níveis adequados em todos os pacientes. À exceção de um paciente, em todos os outros, nos quais a medicação falhou em determinar melhora na oxigenação, ocorreu o óbito. Do mesmo modo, a presença de asfixia perinatal e acidose metabólica grave com necessidade de alcalinização foram variáveis determinantes de mau prognóstico.

No presente momento, o óxido nítrico permanece sendo o método de escolha para o tratamento da HPPRN, em decorrência da excelente resposta apresentada em termos de vasodilatação pulmonar na maioria dos casos tratados e do baixo índice de efeitos colaterais, sobretudo no que diz respeito à preservação da pressão arterial sistêmica. No entanto, estudos recentes têm demonstrado que os inibidores da fosfodiesterase são uma alternativa para o tratamento da HPPRN, podendo inclusive aprimorar a resposta induzida pelo óxido nítrico. Ressalta-se que o tratamento da HPPRN é multifatorial e envolve a necessidade do cuidar das condições precipitantes e/ou que acompanham o quadro. No grupo de pacientes aqui apresentados, em decorrência da indisponibilidade do uso de óxido nítrico, os inibidores da fosfodiesterase consistiram em coadjuvantes importantes para o tratamento da HPPRN grave. Os resultados obtidos sugerem que a redução no índice de oxigenação em uso de milrinona foi um dos fatores determinantes do bom prognóstico. O índice de falha no tratamento da HPPRN, com conseqüente mortalidade encontrada em nossa série $(25 \%)$ foi semelhante ao observado com o uso do óxido nítrico (cerca de 30\%) ${ }^{(41)}$. 


\section{Referências bibliográficas}

1. Hageman JR, Adams MA, Gardner TH. Persistent pulmonary hypertension of the newborn: Trends in incidence, diagnosis and management. Am J Dis Child 1984;38:592-5.

2. Steinhorn $\mathrm{RH}$, Millard SL, Moran FC $3^{\text {rd }}$. Persistent pulmonary hypertension of the newborn: role of nitric oxide and endothelin in pathophysiology and treatment. Clin Perinatol 1995;22:405-28.

3. Walsh MC, Stork EK. Persistent pulmonary hypertension of the newborn. Clin Perinatol 2001;28:609-27.

4. Travadi JN, Patole SK. Phosphodiesterase inhibitors for persistent pulmonary hypertension of the newborn: a review. Pediatr Pulmonol 2003;36:529-35.

5. Weinberger B, Weiss K, Heck DE, Laskin DL, Laskin JD. Pharmacologic therapy of persistent pulmonary hypertension of the newborn. Pharmacol Ther 2001;89:67-79.

6. Konduri GG, Solimano A, Sokol GM, Singer J, Ehrenkranz RA, Singhal N et al. A randomized trial of early versus standard inhaled nitric oxide therapy in term and near-term newborn infants with hypoxic respiratory failure. Pediatrics 2004;113:559-64.

7. Clark RH, Kueser TJ, Walker MW, Southgate WM, Huckaby JL, Peres JA. Low-dose nitric oxide therapy for persistent pulmonary hypertension of the newborn. N Engl J Med 2000;342:469-74.

8. Davidson D, Barefield E, Kattwinkel J, Dudell G, Damask M, Straube R et al. Inhaled nitric oxide for the early treatment of persistent pulmonary hypertension of the term newborn; a randomized double-masked, placebo-controlled dose-response, multicenter study, the I-NO/PPHN Study Group. Pediatrics 1998;101:325-34.

9. Mercier JC, Lacaze T, Storme L, Rozé JC, Dinh-Xuan AT, Dehan M. Diseaserelated response to inhaled nitric oxide in newborns with severe hypoxemic respiratory failure. French Paediatric Study Group of Inhaled NO. Eur J Pediatr 1998;157:745-52.

10. The Neonatal Inhaled Nitric Oxide Study Group. Inhaled nitric oxide in fullterm and nearly full-term infants with hypoxic respiratory failure. N Engl J Med 1997;336:597-604.

11. Ream RS, Hauver JF, Lynch RE, Kountzman B, Gale GB, Mink RB. Lowdose inhaled nitric oxide improves the oxygenation and ventilation of infants and children with acute, hypoxemic respiratory failure. Crit Care Med 1999;27:989-96.

12. Roberts JD Jr, Fineman JR, Morin FC $3^{\text {rd }}$, Shaul PW, Rimar S, Schreiber MD et al. Inhaled nitric oxide and persistent pulmonary hypertension of the newborn. N Engl J Med 1997;336:605-10.

13. Lorch SA, Cnaan A, Barnhart K. Cost-effectiveness of inhaled nitric for the management of persistent pulmonary hypertension of the newborn. Pediatrics 2004;114:417-26.

14. Walsh-Sukys MC, Tyson JE, Wright LL, Bauer CR, Korones SB, Stevenson DK et al. Persistent pulmonary hypertension of the newborn in the era before nitric oxide: practice variation and outcomes. Pediatrics 2000;105:14-20.

15. Findlay RD, Taeusch HW, Walther FJ. Surfactant replacement therapy for meconium aspiration syndrome. Pediatrics 1996;97:48-52.

16. Rais-Bahrami K, Rivera O, Seale WR, Short BL. Effect of nitric oxide in meconium aspiration syndrome after treatment with surfactant. Crit Care Med 1997;25:1744-7.

17. Ambalavanan N, Carlo WA. Hypocapnia and hypercapnia in respiratory management of newborn infants. Clin Perinatol 2001;28:517-31.

18. Sakurai Y, Azarow K, Cutz E, Messineo A, Pearl R, Bohn D. Pulmonary barotrauma in congenital diaphragmatic hernia: a clinicopathological correlation. J Pediatr Surg 1999;34:1813-7.

19. Wung JT, James LS, Kilchevsky E, James E. Management of infants with severe respiratory failure and persistence of fetal circulation, without hyperventilation. Pediatrics 1985;76:488-94

20. Engle WA, Yoder MC, Andreoli SP, Darragh RK, Langefeld CD, Hui SL. Controlled prospective randomized comparison of high frequency jet ventilation and conventional ventilation in neonates with respiratory failure and persistent pulmonary hypertension. J Perinatol 1997;17:3-9.
21. Wiswell TE, Foster NH, Slayter MV, Hachey WE. Management of a piglet model of the meconium aspiration syndrome with high-frequency or conventional ventilation. Am J Dis Child 1992;146:1287-93.

22. Kinsella JP, Truog WE, Walsh WF, Goldberg RN, Bancalari E, Mayock DE et al. Randomized, multicenter trial of inhaled nitric oxide and high-frequency oscillatory ventilation in severe, persistent pulmonary hypertension of the newborn. J Pediatr 1997;131:55-62.

23. Kinsella JP, Abman SH. Controversies in the use of inhaled nitric oxide therapy in the newborn. Clin Perinatol 1998;25:203-17.

24. Barrington KJ, Finner NN. Inhaled nitric oxide for respiratory failure in preterm infants. Cochrane Database Syst Rev 2001;4:CD000399.

25. Chandran S, Haqueb ME, Wickramasinghe HT, Wint Z. Use of the magnesium sulphate in severe persistent pulmonary hypertension of the newborn. J Trop Pediatr 2004;50:219-23.

26. Wu TJ, Teng RJ, Tsou KI. Persistent pulmonary hypertension of the newborn treated with magnesium sulfate in premature neonates. Pediatrics 1995;96:472-4

27. Tolsa JF, Cotting J, Sekarski N, Payot M, Micheli JL, CalameA. Magnesium sulphate as an alternative and safe treatment for severe persistent pulmonary hypertension of the newborn. Arch Dis Child Fetal Neonatal Ed 1995;72:F184-7.

28. Patole S, Lee J, Buettner P, Whitehall J. Improved oxygenation following adenosine infusion in persistent pulmonary hypertension of the newborn. Biol Neonate 1998;74:345-50.

29. Ng C, Franklin O, Vaidya M, Pierce C, Petros A. Adenosine infusion for management of persistent pulmonary hypertension of the newborn. Pediatr Crit Care Med 2004;5:10-3

30. Kato R, Sato J, Nishino T. Milrinone decreases both pulmonary arterial and venous resistances in the hypoxic dogs. Br J Anaesth 1998;81:920-4.

31. Chang AC, Atz AM, Wernovsky G, Burke RP, Wessel DL. Milrinone: systemic and pulmonary hemodynamic effects in neonates after cardiac surgery. Crit Care Med 1995;23:1907-14.

32. McNamara P, Laique F, Muang-In S, Whyte H. Milrinone improves oxygenation in neonates with severe persistent pulmonary hypertension of the newborn. J Crit Care 2006;21:217-22.

33. Bassler D, Choong K, McNamara P, Kirpalani H. Neonatal persistent pulmonary hypertension treated with milrinone: four case reports. Biol Neonate 2006;89:1-5.

34. Shekerdemian LS, Ravn HB, Penny DJ. Intravenous sildenafil lowers pulmonary vascular resistance in a model of neonatal pulmonary hypertension. Am J Respir Crit Care Med 2002;165:1098-102.

35. Sastry BK, Narasimhan C, Reddy NK, Raju BS. Clinical efficacy of sildenafil in primary pulmonary hypertension: a randomized, placebo-controlled, doubleblind, crossover study. J Am Coll Cardiol 2004;43:1149-53.

36. Michelakis WE, Tymchak W, Lien D, Webster L, Hashimoto K, Archer S. Oral sildenafil in an effective and specific pulmonary vasodilator in patients with pulmonary arterial hypertension. Circulation 2002;105:2398-403.

37. Lepore JJ, Maroo A, Pereira NL, Ginns LC, Dec GW, Zapol WM et al. Effect of sildenafil on the acute pulmonary vasodilator response to inhaled nitric oxide in adults with primary pulmonary hypertension. Am J Cardiol 2002;90:677-80.

38. Moreno L, Losada B, Cogolludo A, Lodi F, Lugnier C, Villamor E et al. Postnatal maturation of phosphodiesterase 5 (PDE5) in piglet pulmonary arteries: activity, expression, effects of PDE5 inhibitors and role of the nitric oxide/cyclic GMP pathway. Pediatr Res 2004;56:563-70.

39. Marsh CS, Marden B, Newsom R. Severe retinopathy of prematurity (ROP) in a premature baby treated with sildenafil acetate (Viagra) for pulmonary hypertension. Br J Ophthalmol 2004;88:306-7.

40. Lobato EB, Beaver T, Muehlschlegel J, Kirby DS, Klodell C, Sidi A. Treatment with phosphodiesterase inhibitors type III and V: milrinone and sildenafil is an effective combination during thromboxane-induced acute pulmonary hypertension. Br J Anaesth 2006;96:317-22.

41. Goldman AP, Tasker RC, Haworth SG, Sigston PE, Macrae DJ. Four patterns of response to inhaled nitric oxide for persistent pulmonary hypertension of the newborn. Pediatrics 1996;98:706-13 